\title{
Infraestructura portuaria en Colombia: asimetrías entre el puerto de Buenaventura y el puerto de Cartagena para el año 2015
}

Port Infrastructure in Colombia: Asymmetries between Buenaventura's Port and Cartagena's Port for the Year 2015

\section{Infraestrutura portuária na Colômbia: assimetrias entre o porto de Buenaventura e o porto de Cartagena para o ano 2015}

\author{
Ofelia Castro Castell \\ Elkin David Soler Niño ${ }^{*}$ \\ Ramón Santiago Umaña Castellanos ${ }^{* * *}$ \\ Cristian Yepes Lugo ${ }^{* \star \star x}$
}

Fecha de recibido: 15 de abril de 2016

Fecha de aprobado: 12 de julio de 2016

Doi: http://dx.doi.org/10.12804/http://revistas.urosario.edu.co/index.php/empresa/ article/view/4788

Para citar este artículo: Castro Castell, O., Soler Niño, E. D., Umaña Castellanos, R. S., \& Yepes Lugo, C. (2017). Infraestructura portuaria en Colombia: asimetrías entre el puerto de Buenaventura y el puerto de Cartagena para el año 2015. Universidad \& Empresa, 19132), 87-106. Doi: http://dx.doi.org/10.12804/http://revistas. urosario.edu.co/index.php/empresa/article/view/4788

* Estudiante doctorado en ciencias sociales, Universidad de Buenos Aires UBA. Magíster en gestión de organizaciones, Universidad Militar Nueva Granada. Magíster en comercio internacional y logística integral, Universidad Camilo José Cela. Especialista en gerencia en comercio internacional, Universidad Militar Nueva Granada. Abogada, Universidad Industrial de Santander. Docente-investigador facultad de ciencias económicas y sociales, Universidad de la Salle.Correo electrónico: ofcastro@unisalle.edu.co

** Estudiante décimo semestre de Negocios y Relaciones Internacionales, Universidad de la Salle. Pasante de investigación Semillero Se-koiné facultad de ciencias económicas y sociales, Universidad de la Salle. Correo electrónico: esoler32@unisalle.edu.co

*** Estudiante décimo semestre de Negocios y Relaciones Internacionales, Universidad de la Salle. Pasante de investigación Semillero Se-koiné facultad de ciencias económicas y sociales, Universidad de la Salle. Correo electrónico: rumana46@unisalle.edu.co

**** Doctorando en industria y organizaciones, Universidad Nacional de Colombia. Magíster en Relaciones y Negocios internacionales, Universidad Militar Nueva Granada. Administrador Público, ESAP. Lic. en Filología e Idiomas, Universidad Nacional de Colombia. Docente-investigador facultad de ciencias económicas y sociales, Universidad de la Salle. Correo electrónico: cyepes@unisalle.edu.co 


\section{RESUMEN}

Las dinámicas económicas de las décadas de los ochenta y noventa, junto a lo que ha transcurrido del siglo XXI, han configurado nuevos intereses y relaciones comerciales, impactando, entre otras cosas, las rutas del comercio marítimo. El presente trabajo investigativo tiene como objetivo ofrecer un diagnóstico del estado infraestructural del puerto de Buenaventura en comparación con el puerto de Cartagena, realizando una rigurosa búsqueda de datos informativos, estadísticas e informes para poder definir las asimetrías entre ambos puertos. Los resultados encontrados indican una evidente falta de soporte estatal y desorganización en los procesos internos del manejo del puerto de Buenaventura, el cual, en definitiva, no cuenta con la suficiente capacidad de recepción y despacho de mercancías, lo que genera un aumento en tiempo y en los costos operacionales en los procesos de exportación e importación. El puerto de Buenaventura está por debajo del nivel infraestructural del puerto de Cartagena.

Palabras clave: Puerto, Buenaventura, Cartagena, infraestructura, logística comercial, comercio marítimo.

\section{ABSTRACT}

The economic dynamics of the 80 s and 90 s decade, with what has passed twenty-first century, have set new interests and trade relations, impacting, among other things, maritime trade routes. This research work aims to provide a diagnosis of infrastructural Buenaventura port status compared with the port of Cartagena, performing a thorough search of informative data, statistics, and reports; to define the asymmetries between the two ports.

The results indicate a clear lack of state support and disorganization in the internal processes of management of the port of Buenaventura, which, ultimately, does not have sufficient capacity to receive and dispatch goods, which generates an increase in time and in operating costs in export and import processes. Buenaventura port infrastructure is below the level of the port of Cartagena.

Key Words: Port, Buenaventura, Cartagena, infrastructure, trade logistics, maritime trade.

\section{RESUMO}

As dinâmicas económicas das décadas dos 80 e 90, junto ao que tem transcorrido do século XXI, têm configurado novos interesses e relações comerciais, impactando, entre outras coisas, as rotas do comércio marítimo. O presente trabalho investigativo tem como objetivo oferecer um diagnóstico do estado infraestrutural do porto de Buenaventura comparando-o com o porto de Cartagena, realizando uma rigorosa busca de dados informativos, estáticas, informes, para conseguir definir as assimetrias entre ambos os portos.

Os resultados encontrados indicam uma evidente falta de suporte estatal e desorganização nos processos internos da gestão do porto de Buenaventura, o qual, em definitiva, não conta com a suficiente capacidade de recepção e despacho de mercancias, o qual gera um aumento em tempo e nos custos operacionais nos processos de exportação e importação. O porto de Buenaventura está por debaixo do nível infraestrutural do porto de Cartagena.

Palavras-chave: Porto, Buenaventura, Cartagena, infraestrutura, logística comercial, comércio marítimo. 


\section{INTRODUCCIÓN}

El desarrollo económico a finales del siglo pasado estuvo inmerso en dinámicas de cooperación que permitieron la apertura de mercados a nivel global; esto contribuyó a que los países generaran estrategias con el fin de superar las devastaciones de la segunda guerra mundial y el comercio se convirtió en la solución más diplomática e incluyente para alcanzar este objetivo. En el caso específico de Colombia, a principios de la década de los noventas el país abrió de manera activa sus barreras comerciales.

A partir de ese momento el país entra en una dinámica económica muy vertiginosa, que hace importante la focalización en el desarrollo de la infraestructura. La firma de tratados de libre comercio y otros acuerdos comerciales empujan al país a buscar soluciones ante su falta de infraestructura comercial, por lo que se hace fundamental ampliar la capacidad y mejorar la efectividad que hay en sus puertos, especialmente en los puertos que tienen una localización geográfica estratégica, como lo es el puerto de Buenaventura.

\section{METODOLOGÍA}

El presente trabajo investigativo tiene un enfoque cualitativo. Para ofrecer al lector el diagnóstico del estado infraestructural del puerto de Buena- ventura, se mostrarán las características de dicho puerto, para después, a través de un símil (método comparativo), realizar el respectivo análisis respecto a las características del puerto de Cartagena. Esto, dado que una investigación de la CEPAL para 2014, clasificó el puerto de Cartagena como el más importante de Colombia (CEPAL, 2014); también, en este mismo estudio se establece un ranking que cataloga este puerto como el mejor puerto comercial del país, clasificándolo en el puesto 5 de los 120 puertos comerciales evaluados de Latinoamérica, mientras que el puerto de Buenaventura fue clasificado en el puesto 17. Es por esto que el puerto de Cartagena aparece como el mejor punto de comparación, ya que, además de estar evidentemente mejor posicionado, está situado en el mismo país y por lo tanto, en un mismo contexto.

Debido a que la investigación va dirigida al desarrollo infraestructural del puerto de Buenaventura, la región en la que se ubica con respecto a su par comparado es importante, pero no fundamental. Ambos son los puertos más importantes de sus regiones, sin embargo no se está haciendo un análisis con respecto a la ubicación de los puertos y su posible impacto, sino a partir de su importancia y su estado infraestructural. El proceso de recolección de datos inició con la investigación del 
panoramacomercialdelpaís, paraentender sus procesos comerciales a lo largo del tiempo y el tipo de relaciones comerciales con las cuales se estaba vinculando, así como los medios de transporte y su eficiencia en cuanto a los despachos comerciales. Se recolectaron datos estadísticos sobre la condición infraestructural que los puertos han tenido a lo largo del tiempo, su gestión administrativa, mejoras y obstáculos.

Finalmente, los datos se clasificaron según su importancia, generando un análisis comparativo del estado infraestructural de los puertos, sus ventajas, desventajas y desafíos teniendo como premisa el contexto global y nacional que enfrenta Colombia en materia de comercio marítimo y relaciones comerciales, para así, presentar unos resultados fundamentados desde una perspectiva actual con un soporte importante de datos y recolección de información histórica con respecto a conformación y desarrollo de los puertos. Con esto se busca evidenciar la carencia de infraestructura portuaria que tiene el puerto de buenaventura, a pesar de su preponderancia como zona geoestratégica en términos comerciales.

\section{MARCO TEÓRICO}

El desarrollo económico y los factores de competitividad de un país, están ligados a la infraestructura y al sistema logístico que este desarrolle. Tal como lo plantea Snieska y Draksaite (2007), las condiciones de la globalización llevan a que para cada país sea muy importante aumentar el nivel de competitividad de su economía, determinado por la evaluación de varios indicadores: la infraestructura, el macroambiente, la actividad y la intensidad de los mercados, innovaciones y otros. Poseer una buena infraestructura logística, significa estar verdaderamente abiertos para los negocios.

Los sistemas logísticos requieren la sincronización de los actores participantes (Johnson, Wood, Wardlow \& Murphy, 1999), es posible entonces determinar una constante interconexión entre los elementos de los sistemas logísticos: transporte, los procesos y la información (Navickas, Sujeta \& Vojtovich, 2011). Por ejemplo, la efectividad en el transporte aumentará el flujo del comercio, debido a que permite reducir costos y tiempo.

La industria logística en Colombia tiene un alto potencial de desarrollo, debido a que las limitaciones que aún presenta son demasiadas, habiendo mucho por hacer. Colombia tiene una excelente posición geográfica, lo que le permite tener puertos en el Atlántico y el Pacífico; sin 
embargo, la puesta en marcha de un modelo económico que por mucho tiempo fue proteccionista, la burocracia y la falta de inversión en infraestructura han tenido como consecuencia el desaprovechamiento de esa condición natural.

La historia portuaria en Colombia se origina desde los tiempos de la conquista y el descubrimiento de América, donde su desarrollo estuvo dirigido por los intereses de la Corona española. Las principales rutas de comercio iniciaron en la región atlántica del país, dando origen a nuevas ciudades como Santa Marta y Cartagena, en las que, años después, se establecen rutas marítimas específicas que le dieron preponderancia a la zona comercial de Cartagena, convirtiéndose en el principal puerto de Sudamérica en el tráfico de mercancías hacia Europa, comerciando, oro, esclavos y otro tipo de riquezas adquiridas durante este periodo. (Rendón, 2009).

Tiempo después, la demanda comercial se fue acrecentando y los puertos comenzaron a ser administrados por compañías privadas y entidades públicas de carácter regional, los cuales dieron paso a nuevos puertos y vías de comercialización; esta expansión permitió el desarrollo de nuevas formas de fortalecimiento económico para el país.
Sin embargo, el crecimiento industrial y las nuevas demandas expusieron la falta de infraestructura y gestión que existía por parte de los administradores de los puertos. "los puertos presentaron serias dificultades por falta de personal, poca preparación, escasos medios técnicos, mínimos recursos económicos, muelles insuficientes, etc. Era evidente que los puertos no respondían a las necesidades reales del país, y no estaban adecuados para atender el pedido, cada vez mayor, de servicios para barcos y carga" (Rendón, 2009, p. 100)

Por esta razón se creó la empresa de "Puertos de Colombia" en 1959, la cual buscaba ser la primera entidad autónoma con suficiente solvencia y liquidez para manejar los puertos encargándose de todo el proceso de mejoramiento industrial y logístico. A través de los años, la nueva entidad buscó fomentar la formación de los individuos para mejorar los procesos internos en los puertos; sin embargo, la empresa Puertos de Colombia no logró implementar este tipo de formación del trabajador portuario.

"Con los recursos del Banco Mundial, se brindó capacitación a los trabajadores, pero no se desarrollaron los procesos de formación por competencias y evaluación del desempeño y finalmente, el poco compromiso 
de los trabajadores para salvar a la empresa, la llevó a su liquidación" (Rendón, 2009, p.101). Además, las inversiones en los puertos no eran suficientes debido a que no se destinaban a las necesidades que cada puerto demandaba según el volumen de comercio exterior que manejasen.

Para ese momento, Buenaventura movía el 50\% del comercio exterior colombiano, Santa Marta el 20\% y Cartagena, Barranquilla y Tumaco el 30\% restante. Luego, en 1991, se puso en marcha la reforma portuaria en Colombia y se dio un cambio radical en la situación de los puertos. Se liquidó la empresa de Puertos de Colombia (Colpuertos) y, en cambio, se crearon la Superintendencia General de Puertos y las Sociedades Portuarias; además, se dio la conformación de empresas de operadores portuarios privados. (Ministerio de Transporte, 2014).

Entre los objetivos de esta Ley están la abolición del monopolio estatal de los puertos y la entrega de estos en concesión, la modernización de los mismos y el mejoramiento de su eficiencia. "Ése fue el principio del cambio del sistema portuario y del comercio marítimo en Colombia y con este esquema de privatización, la Nación empezó a percibir ingresos por el uso de sus recursos costeros y de las infraestructuras portuarias que durante años estuvo construyendo y manteniendo" (Ministerio de Transporte, 2014, p.1).

La nueva organización institucional buscaba redefinir la función del Estado y eliminar el monopolio de los puertos. Además de esto, la apertura económica de la época permitió que las empresas privadas funcionaran como un soporte de recursos para el desarrollo de los puertos y el Estado comienza a cumplir una función en la distribución y planificación de las concesiones que otorgaba al sistema portuario colombiano.

Después de ese periodo de reorganización normativa en 1991 se expidió el "Estatuto de Puertos Marítimos (Ley 01 de 1991) y sus decretos reglamentarios, los Ministerios de Transporte y Medio Ambiente los cuales tienen la responsabilidad de planificar y racionalizar el desarrollo de la actividad portuaria en puertos marítimos, con la finalidad de hacerla más eficiente y competitiva." (Departamento Nacional de Planeación, 2001, p. 2).

Además de esto, la última década del siglo pasado trajo consigo la apertura económica con la firma del Consenso de Washington en 1991, donde se implementaron nuevas políticas económicas con enfoque neoliberal, con el fin de proyectar 
la economía colombiana ante las nuevas dinámicas del sistema mundial. A partir de la implementación de estas políticas se inició en Colombia una expansión en el ámbito del comercio internacional la cual trajo la firma de nuevos acuerdos comerciales entre países vecinos y nuevas economías desarrolladas.

En la actualidad, Colombia ha logrado firmar acuerdos con países como México, el 13 junio de 1994; el Salvador, suscrito en Marzo de 2010; Nicaragua, firmado en 1985; Honduras, en Febrero de 2010; Guatemala, en noviembre de 2009; Canadá, firmado en Diciembre de 2009; Estados Unidos, firmado en Noviembre 2006; Cuba, en Julio de 2001; Chile, con el cual firmó un tratado que entró en vigor en mayo del 2009 , y la Unión Europea, en junio de 2012. El país también ha participado de acuerdos como el suscrito con la CAN (ComunidadAndina) ${ }^{1}$ en 1969 , y que ha tenido varias modificaciones a lo largo del tiempo, siendo uno de los primeros acuerdos firmados por Colombia; El de la EFTA, suscrito en noviembre de 2008; MERCOSUR, suscrito el 8 de Octubre de 2004, y CARICOM, firmado el 24 de Julio de 1994, el cual ha tenido unos balances muy positivos para todos los miembros, principalmente para Colombia; este acuerdo se firmó con 12 Estados de la región pacífica. (Ministerio de Industria y Turismo, 2014).

Estos nuevos acuerdos han tenido impactos tanto negativos como positivos. Han permitido obtener nuevos productos y competencias que se pueden implementar para mejorar los procesos internos; sin embargo la escasa infraestructura interna, portuaria y de comunicación del país no ha permitido que Colombia se beneficie como debería de estos acuerdos, ya que las demandas infraestructurales y tecnológicas que traen consigo la firma de estos acuerdos no han podido ser solventadas, por lo que el país se ve rezagado con respecto a sus pares; el mejoramiento de los puertos y sus vías de comunicación y canales de distribución se convierten en una necesidad y prioridad en la agenda económica.

Actualmente, en Colombia la infraestructura portuaria es aceptable, más no suficiente para solventar la demanda mercantil. Colombia se clasifica en el puesto 93 (entre 189 economías evaluadas) en las operaciones de comercio transfronterizo. Para conocer el estado en cuanto

1 La Comunidad Andina está compuesta por Perú, Bolivia, Colombia y Ecuador. 
a los procesos de comercialización (importación y exportación), es decir la efectividad que tiene Colombia con respecto a sus pares en Latinoamérica y la OCDE se presenta la siguiente tabla con información relevante que permitirá entender el estado de la logística portuaria ligada a sus fallas infraestructurales.

Tabla 1. Comercio Transfronterizo Colombia

\begin{tabular}{|l|c|c|c|}
\hline \multicolumn{1}{|c|}{ Indicador } & Colombia & $\begin{array}{c}\text { América Latina } \\
\text { y Caribe }\end{array}$ & OCDE \\
\hline Tiempo para importar: Cumplimiento fronterizo (horas) & 112 & 86 & 15 \\
\hline Costo para importar: Cumplimiento fronterizo (USD) & 545 & 493 & 160 \\
\hline Tiempo para importar: Cumplimiento documental (horas) & 64 & 68 & 5 \\
\hline Costo para importar: Cumplimiento documental (USD) & 50 & 134 & 36 \\
\hline Tiempo para exportar: Cumplimiento fronterizo (horas) & 112 & 107 & 9 \\
\hline Costo para exportar: Cumplimiento fronterizo (USD) & 545 & 665 & 123 \\
\hline Tiempo para exportar: Cumplimiento documental (horas) & 60 & 93 & 4 \\
\hline Costo para exportar: Cumplimiento documental (USD) & 90 & 128 & 25 \\
\hline
\end{tabular}

Fuente: elaboración propia, basado en Doing Business (2015).

En la tabla se evidencia cómo los costos de importación y exportación, así como el tiempo son superiores al promedio comparado, lo cual impide la entrada de mercancías de forma más frecuente y frena la expansión de flujo comercial, debido a las falencias que presentan los puertos; esto respalda el planteamiento sobre la carencia de infraestructura portuaria en Colombia como una problemática general y no solo en algunas regiones específicas, puesto que los altos costos representan una disminución en el interés de desarrollar relaciones comerciales equitativas.
A través de los años, Colombia tuvo un sistema de desarrollo e industrialización a través de la captación de divisas como fuente de recursos para financiar este crecimiento; las divisas eran obtenidas de la venta de café, siendo este el recurso más demandado en el mercado global. Sin embargo, las fuertes crisis financieras y de mercado en el siglo pasado causaron un impacto negativo en la economía, lo cual llevó a que se implementaran una serie de medidas proteccionistas a través de aranceles, control directo sobre las importaciones y el flujo de divisas (Banco de La Republica, 2014). 
Con el fin de superar los obstáculos presentados se implementaron políticas de desarrollo planificadas para generar una producción localizada fundamentada en la sustitución de importaciones, buscando fortalecer las economías de América Latina. El proceso fue favorable para las industrias nacionales; no obstante, las dinámicas internacionales estaban cambiando y era necesario adoptar nuevas medi- das, por esto se firmaron nuevos acuerdos comerciales y políticos en los cuales el ámbito comercial tuviese un rol preponderante en la economía (Banco de La Republica, 2014).

En las siguientes tablas se mostrará la evolución del comercio y su importancia en la economía, haciendo énfasis en los procesos de importación y exportación.

Tabla 2. Colombia - Exportaciones de Mercancías

\begin{tabular}{|c|c|c|}
\hline \multicolumn{3}{|c|}{ Colombia - Exportaciones de Mercancías } \\
\hline Año & Exportaciones (Dólares) & Exportaciones \% del PIB \\
\hline 2014 & 54.828 millones & 14,52 \\
\hline 2013 & 58.821 millones & 15,48 \\
\hline 2012 & 60.273 millones & 16,31 \\
\hline 2011 & 57.420 millones & 17,11 \\
\hline 2010 & 39.819 millones & 13,89 \\
\hline 2009 & 32.853 millones & 14,03 \\
\hline 2008 & 37.626 millones & 15,43 \\
\hline 2007 & 29.991 millones & 14,46 \\
\hline 2006 & 24.390 millones & 15 \\
\hline 2005 & 21.190 millones & 14,48 \\
\hline 2004 & 16.223 millones & 13,85 \\
\hline 2003 & 13.079 millones & 13,81 \\
\hline 2002 & 11.911 millones & 12,15 \\
\hline 2001 & 12.290 millones & 12,52 \\
\hline
\end{tabular}

Fuente: elaboración propia, basado en DatosMacro (2014). 
Tabla 3. Colombia - Importación de Mercancías

\begin{tabular}{|l|c|c|}
\hline \multicolumn{3}{|c|}{ Colombia - Importación de Mercancías } \\
\hline Fecha & Importaciones (Dólares) & Importaciones \% PIB \\
\hline 2014 & $64.029,0$ Millones & $16,96 \%$ \\
\hline 2013 & $59.397,0$ Millones & $15,63 \%$ \\
\hline 2012 & $59.111,4$ Millones & $16,00 \%$ \\
\hline 2011 & $54.674,8$ Millones & $16,29 \%$ \\
\hline 2010 & $40.682,7$ Millones & $14,19 \%$ \\
\hline 2009 & $32.897,7$ Millones & $14,05 \%$ \\
\hline 2008 & $39.668,8$ Millones & $16,27 \%$ \\
\hline 2007 & $32.897,0$ Millones & $15,86 \%$ \\
\hline 2006 & $26.162,3$ Millones & $16,09 \%$ \\
\hline 2005 & $21.204,4$ Millones & $14,49 \%$ \\
\hline 2004 & $16.745,7$ Millones & $14,30 \%$ \\
\hline 2003 & $13.888,5$ Millones & $14,66 \%$ \\
\hline 2002 & $12.711,1$ Millones & $12,96 \%$ \\
\hline 2001 & $12.834,0$ Millones & $13,07 \%$ \\
\hline
\end{tabular}

Fuente: elaboración propia, basado en DatosMacro (2014).

Es posible observar que, en ambos casos, el aumento paulatino de los proceso comerciales tiene cada vez una relevancia mayor en la económica colombiana, debido a los nuevos acuerdos y a las demandas que exigen estos acuerdos. En cuanto a la distribución de las mercancías en los principales puertos de Colombia, el puerto de Buenaventura tiene una participación considerable con respecto a sus pares.

\section{PUERTO DE BUENAVENTURA}

\subsection{Sociedad Portuaria Regional de Buenaventura S.A.}

La Sociedad Portuaria Regional de Buenaventura S.A. es una empresa de economía mixta, regida por el derecho privado. El $83 \%$ de su participación accionaria pertenece a empresarios privados conformados por importadores, exportadores, operadores portuarios, líneas navieras, 
gremios, ex trabajadores portuarios y personas naturales. El 15\% restante está en manos del sector público integrado por la Alcaldía de Buenaventura y el $2 \%$ para el Ministerio de Transporte (Sociedad Portuaria Regional de Buenaventura, s.f.).

El puerto está localizado en la Costa Pacífica al sur-occidente de Colombia. Se encuentra ubicado cerca al canal de Panamá, equidistante entre los puertos de Vancouver y Valparaíso, y tiene una posición geoestratégica muy importante por su cercanía con las principales rutas marítimas del pacífico, que atraviesan el planeta de norte a sur y de oriente a occidente (Procolombia, 2015).

El canal de acceso al puerto de Buenaventura presenta dos inconvenientes. Por un lado, se presentan altas de sedimentación por el aporte de sedimentos de los ríos y de la deriva litoral. De otro lado, un inconveniente es que bajo el fondo de sedimentos sueltos se encuentra una roca limolita, que presenta dificultades técnicas para su retiro. Se requieren labores de mantenimiento continuo (Grupo de Planificación Sectorial, 2011).

Su tipo de puerto es multipropósito marítimo, tiene una extensión de 620 hectáreas y cuenta con 250 empleados. El acceso al puerto puede ser por camión y/o ferrocarril y las distancias respecto a las principales ciudades del país son: Bogotá, 504 km; Medellín, 498 km, y Cali, 165 km; además, tiene cercanía de $120 \mathrm{~km}$ con zona franca (Procolombia, 2015).

El puerto cuenta con 14 muelles que tienen una longitud en metros lineales de 1.998 y están diseñados bajo la especialidad multipropósito comentada anteriormente. Para el almacenamiento tiene $13.119 \mathrm{~m}$ de bodegas cubiertas, una capacidad para importación y exportación de $27.044 \mathrm{~m}^{2}$, una capacidad de patios de $48.000 \mathrm{~m}$, aproximadamente, una capacidad para granel sólido de 164.000 toneladas y de $230.000 \mathrm{~m}^{3}$ para granel líquido (Procolombia, 2015).

Sus principales equipos son cuatro grúas pórtico y otros equipos para manejo (3 grúas móviles, 16 reach stackers y 4 grúas Pórtico Post-Panamax). Cuenta con 384 tomas de conexión para contenedores refrigerados y con un escáner. Sus navieras son: China Shipping Container Lines, CMA-CGM, Compañía Chilena de Navegación (CCNI), Compañía Sudamericana de Vapores, Evergreen Marine, Hamburg Sud Hapag Lloyd, Kawasaki Kisen Kaisha (K - LINE), Maruba, Mediterranean Shipping y TBS Pacific Liner (Procolombia, 2015). 
Finalmente, los principales productos que maneja son azúcar a granel y en sacos, vehículos, maquinaria y equipo, carbón, hulla, melaza, transformadores, aceites y lubricantes, partes maquinarias, implementos eléctricos, partes, accesorios y repuestos (Procolombia, 2015).

Otros datos del puerto:

Latitud: $3^{\circ} 53^{\prime} 35^{\prime} \mathrm{N}$

Longitud: $77^{\circ} 04^{\prime} 45^{\prime} \mathrm{W}$

Para el acceso al puerto

Longitud: $31.5 \mathrm{~km}$

Profundidad: $10.7 \mathrm{~m}$

Calado máximo en muelles: 41 pies

\section{PUERTO DE CARTAGENA}

\subsection{Sociedad Portuaria Regional de Cartagena S.A.}

Debido al cambio en el manejo de los puertos en Colombia, la Sociedad Portuaria Regional de Cartagena maneja la misma estructura en la participación accionaria, donde alrededor del $80 \%$ corresponde a entes privados; la diferencia es que la concesión otorgada aeste puerto fue por 40 años, es decir, es más longeva que la concesión del puerto de Buenaventura (20 años).

Es reconocido como un gran puerto logístico y de transbordo internacional especializado en el comercio de contenedores (Procolombia, 2015) está localizado en el Caribe colombiano en la parte norte del país y tiene gran relevancia debido a su cercanía con el canal de Panamá.

El puerto tiene limitaciones en términos de profundidad y ancho, además, en la zona de influencia del canal se localizan los fuertes de San Fernando y San José, por lo que es prioritario que, ante cualquier intervención en el canal, se considere la estabilidad y conservación de dichos monumentos, ya que son patrimonio histórico y cultural de la humanidad. Hasta el momento no se presentan problemas de sedimentación, por lo que el mantenimiento se puede enfocar sólo a la protección de los fuertes (Grupo de Planificación Sectorial, 2011).

El puerto de Cartagena es de tipo marítimo con acceso fluvial a través del Canal del Dique. La extensión del puerto es de 40 hectáreas, cuenta con 700 empleados (200 directos y 500 indirectos) y sólo se puede acceder a él por camión. Las distancias de este respecto a las principales ciudades del paísson: Bogotá, $1.125 \mathrm{Km}$; Medellín, $632 \mathrm{Km}$, y Cali, $1.012 \mathrm{~km}$. La ventaja del puerto es que en Cartagena hay dos zonas francas: la candelaria (a $10 \mathrm{Km}$ de distancia) y la Zona Franca Comercial (a $300 \mathrm{~m}$ de distancia) (Procolombia, 2015). 
Respecto a los muelles, cuenta con 8 y tienen una longitud en metros lineales de 1.636 con la especialidad en contenedores. Para el almacenamiento tiene 5 bodegas cubiertas, una capacidad para importación y exportación de $30.000 \mathrm{~m}^{2}$, una capacidad de patios de 22 hectáreas, una capacidad para granel sólido de 20.000 toneladas y no maneja granel líquido (Procolombia, 2015).

Sus principales equipos son cuatro grúas pórtico y otros equipos para manejo (2 grúas móviles, 21 Transtainer, 14 reach stackers, 67 plataformas y 62 camiones). Cuenta con 550 tomas de conexión para contenedores refrigerados y con un escáner. Sus navieras son: American President, China Shipping Container Lines, CMA-CGM, Compañía
Chilena de Navegación (CCNI), Compañía Sudamericana de Vapores, Cosco Line, Evergreen Marine, Frontier Liner Services, Hamburg Sud, Hapag Lloyd, Mediterranean Shipping y Seaboard Mairne (Procolombia, 2015).

Por último, los principales productos que maneja el puerto son: manufacturas e insumos básicos y derivados, agroindustria, tradicional, prendas de vestir y servicios de entretenimiento.

Otros datos del puerto:

Latitud: $10^{\circ} 24^{\prime} 9^{\prime} \mathrm{N}$

Longitud: $75^{\circ} 31^{\prime} 12^{\prime} \mathrm{W}$

Para el acceso al puerto:

Longitud: $82 \mathrm{~km}$

Profundidad: $15 \mathrm{~m}$

Calado máximo en muelles: 43 pies

\section{ANÁLISIS COMPARATIVO}

Tabla 4. Cuadro comparativo de la infraestructura portuaria

\begin{tabular}{|l|c|c|}
\hline & Puerto Buenaventura & Puerto Cartagena \\
\hline Extensión del puerto & 620 Hectáreas & 40 Hectáreas \\
\hline Número de empleados & 250 & 700 \\
\hline Distancias terrestres principales ciudades $(\mathrm{km})$ & 504 & 1.125 \\
\hline Bogotá & 498 & 632 \\
\hline Medellín & 165 & 1.012 \\
\hline Cali & $120 \mathrm{~km}$ & $10 \mathrm{~km}$ y $300 \mathrm{~m}$ \\
\hline Proximidad Zona Franca & & \\
\hline
\end{tabular}




\begin{tabular}{|c|c|c|}
\hline & Puerto Buenaventura & Puerto Cartagena \\
\hline \multicolumn{3}{|l|}{ Para acceso al puerto } \\
\hline Longitud & $31,5 \mathrm{~km}$ & $82 \mathrm{~km}$ \\
\hline Profundidad & $10,7 \mathrm{~m}$ & $15 \mathrm{~m}$ \\
\hline Calado máximo en Muelles & 41 pies & 43 pies \\
\hline \multicolumn{3}{|l|}{ Muelles } \\
\hline Número & 14 & 8 \\
\hline Longitud en metros lineales & $1.998 \mathrm{~m}$ & $1.636 \mathrm{~m}$ \\
\hline \multicolumn{3}{|l|}{ Almacenamiento } \\
\hline $\begin{array}{l}\text { Capacidad para importación y } \\
\text { exportación }\end{array}$ & $27.044 \mathrm{~m}^{2}$ & $30.000 \mathrm{~m}^{2}$ \\
\hline Capacidad Patios & $48.000 \mathrm{~m}$ aprox. & 22 Hectáreas \\
\hline Capacidad Granel Sólido & 164.000 toneladas & 20.000 ton (dato 2014) \\
\hline \multicolumn{3}{|l|}{ Principales equipos } \\
\hline Grúas Pórtico & 4 & 4 \\
\hline \multirow{3}{*}{ Otros Equipos } & 3 Grúas & \multirow{3}{*}{$\begin{array}{l}2 \text { grúas móviles; } 21 \text { Trans- } \\
\text { tainer, } 14 \text { Reach Stacker;67 } \\
\text { plataformas; } 62 \text { Camiones }\end{array}$} \\
\hline & 16 Reach Stackers & \\
\hline & 4 grúas Pórtico Post-Panamax & \\
\hline \multicolumn{3}{|l|}{ Otras Facilidades } \\
\hline $\begin{array}{l}\text { Tomas conexión contenedores } \\
\text { refrigerados }\end{array}$ & 384 & 550 \\
\hline Escáner & 1 & 1 \\
\hline \multicolumn{3}{|l|}{ Rendimiento Portuario } \\
\hline $\begin{array}{l}\text { Promedio rata de cargue } \\
\text { contenedores }\end{array}$ & 31 Buque / Hora & 45 Movimientos/Hora \\
\hline $\begin{array}{l}\text { Promedio tiempo estadía } \\
\text { buques (días) }\end{array}$ & 24 Horas & 12 Horas \\
\hline \multicolumn{3}{|c|}{ Tarifas promedio para carga (impo - expo) } \\
\hline \multirow{2}{*}{$\begin{array}{l}\text { Carga General y Gráneles } \\
\text { (Promedio)/por ton }\end{array}$} & Carga general 1 a 10,000 & \multirow{2}{*}{ Entre US $\$ 4,00$ y US\$4,50 } \\
\hline & toneladas año US\$ 4,70 ton & \\
\hline
\end{tabular}




\begin{tabular}{|c|c|c|}
\hline & Puerto Buenaventura & Puerto Cartagena \\
\hline \multirow{4}{*}{$\begin{array}{l}\text { Contenedores } 20^{\prime} \text { y } 40^{\prime} \text { 'Llenos/ } \\
\text { Unidad }\end{array}$} & Contenedor 20' de 1 a & \multirow{4}{*}{ Entre US\$ 85 y US\$ 115} \\
\hline & $625 /$ año US\$ 80 por Unidad & \\
\hline & Contenedor $40^{\prime}$ de 1 & \\
\hline & a 652/año US\$ 98 por Unidad & \\
\hline \multirow{5}{*}{$\begin{array}{l}\text { Vehículos Entre } 1 \mathrm{~m} 3 \text { y } 40 \mathrm{~m} 3 \\
\text { / Unidad }\end{array}$} & De acuerdo con $\mathrm{M}^{3}$ por Unidad & \multirow{5}{*}{ Entre US\$ 58 y US\$ 140} \\
\hline & Hasta $10 \mathrm{~m}^{3}:$ US\$ 28 & \\
\hline & Hasta $19,9 \mathrm{~m}^{3}$ : US\$ 39 & \\
\hline & Hasta $40 \mathrm{~m}^{3}$ US $\$ 72$ & \\
\hline & Más $\mathrm{m}^{3}$ US\$ 132 & \\
\hline \multicolumn{3}{|l|}{ Para almacenamiento } \\
\hline Días libres & 3 días impo / 5 días expo & 3 \\
\hline \multirow{3}{*}{$\begin{array}{l}\text { En Cubierto: Carga General } \\
\text { Tonelada / Día (Promedio) }\end{array}$} & US\$ 1,40 día/ton hasta el día 5 & \multirow{3}{*}{ US\$ 1,50} \\
\hline & US\$ 1,63 día/ton hasta el día 10 & \\
\hline & US\$ 4,05 día/ton en adelante & \\
\hline \multirow{3}{*}{$\begin{array}{l}\text { Carga General Tonelada / } \\
\text { Día (Promedio) }\end{array}$} & US\$ 1,10 día/ton hasta el día 5 & \multirow{3}{*}{$\begin{array}{c}\text { US\$ } 1,00 \text { hasta el día } 5 \\
\text { US\$ } 1,30 \text { hasta el día } 10 \\
\text { US\$ } 1,80 \text { en adelante }\end{array}$} \\
\hline & US\$ 1,30 día/ton hasta el día 10 & \\
\hline & US\$ 3,15 día/ton en adelante & \\
\hline \multirow{3}{*}{$\begin{array}{l}\text { Contenedor de } 20 \text { ' Lleno } \\
\text { Unidad/Día }\end{array}$} & U\$ $18,15 /$ día, hasta el 4 o 6 día & \multirow{3}{*}{$\begin{array}{c}\text { US\$ } 15 \text { hasta el día } 5 \\
\text { US\$ } 20 \text { hasta el día } 10 \\
\text { US\$ } 25 \text { por día en adelante }\end{array}$} \\
\hline & US\$ 21.45 hasta el 10 día & \\
\hline & US\$ 41,45 por día en adelante & \\
\hline \multirow{3}{*}{$\begin{array}{l}\text { Contenedor de } 40 \text { ' Lleno } \\
\text { Unidad/Día }\end{array}$} & U\$ 20,35/día, hasta el 4 o 6 día & \multirow{3}{*}{$\begin{array}{l}\text { US\$ } 25 \text { hasta el día } 5 \\
\text { US\$ } 30 \text { hasta el día } 10 \\
\text { US\$ } 40 \text { en adelante }\end{array}$} \\
\hline & US\$ 24,05 hasta el 10 día & \\
\hline & US\$ 50,40 por día en adelante & \\
\hline
\end{tabular}

Fuente: elaboración propia, basado en Procolombia (2015) - Sociedades Portuarias Regionales.

En la tabla 4 se evidencia la gran brecha que existe entre los dos puertos. El puerto de Cartagena es mucho más pequeño y sin embargo goza de un mejor estado infraestruc- tural. El puerto de Cartagena tiene 450 empleados más, es decir, está siendo más efectivo a la hora de estimular la economía mediante el factor trabajo. 
La profundidad y el calado son mayores a los del puerto de Buenaventura; esto facilita las operaciones portuarias al minimizar el riesgo de las embarcaciones tanto que entran como que salen.

La capacidad de almacenamiento del puerto de Cartagena para las importaciones y exportaciones sobrepasa la de Buenaventura casi por $3.000 \mathrm{~m}^{2}$ y la brecha en la capacidad de patios es abrumadora. El puerto de Cartagena no sólo cuenta con más equipos principales, sino que además son más tecnificados (caso del transtainer y plataformas) sin contar con la gran diferencia en las conexiones para contenedores refrigerados.

Las falencias descritas anteriormente se ven reflejadas en los índices de rendimiento portuario y los precios. En una hora, el puerto de Cartagena realiza el proceso de cargue a 14 contenedores más que los que realiza el puerto de Buenaventura en el mismo lapso y los despacha 12 horas antes sin inflar demasiado los precios, es decir, el puerto de Cartagena está despachando más contenedores a un menor tiempo y a un precio similar.

Por último, el puerto de Cartagena tiene precios más bajos respecto al almacenamiento, esto gracias a que posee una mayor capacidad frente al puerto de Buenaventura, que le per- mite hacerlo; en pocas palabras, el puerto de Cartagena es mucho más eficiente y competitivo.

"Colombia se caracteriza por contar con un importante número de centros urbanos y productivos en la parte central del territorio. Por esta razón, y a pesar de contar con el privilegio de tener un doble acceso marítimo, la infraestructura [...] debería poder garantizar una rápida y eficiente movilización de los grandes volúmenes de carga" (Pérez, 2005, p. 5). Los tres principales centros urbanos y productivos del país son Bogotá, Cali y Medellín.

El problema es que el puerto de Buenaventura es el más cercano a dichos centros productivos y a los alrededores de estos $\mathrm{y}$, al presentar el rezago infraestructural descrito anteriormente, no sólo representa una barrera frente a la eficiente movilización de los grandes volúmenes de carga, sino que afecta los costos de transacción de forma negativa al hacer que la información en los mercados regionales sobre los excesos de oferta y/o demanda de bienes, no pueda ser aprovechada en forma adecuada (Pérez, 2005).

\section{CONCLUSIONES}

Las tendencias globales comerciales se dirigen hacia los países asiáticos 
por los cuales Colombia tiene acceso directo a través del océano pacifico y el puerto de Buenaventura como un lugar estratégico para llevar a cabo la ampliación de vías y relaciones comerciales.

En la actualidad, la infraestructura portuaria continúa teniendo problemas debido a su incapacidad de cumplir con las demandas comerciales que se están presentando; sin embargo, es importante reconocer que se han hecho mejoras en los puertos, es decir, existe una infraestructura portuaria en buen estado, aunque sigue siendo insuficiente para las nuevas demandas.

Esta problemática también causa fuertes golpes a las industrias nacionales que no cuentan con la tecnología necesaria para competir con grandes empresas comerciales que tienen una gran capacidad de comercialización de sus productos. Los tiempos de distribución de las mercancías que entran y salen país es muy lento y costoso e impide una correcta ejecución de los acuerdos firmados, afectando negativamente la industria comercial nacional.

Como fue posible observar en el documento y las gráficas referidas al proceso de importación, la principal causa de que no exista un sistema de comercio más acorde con las demandas globales son dos factores, el primero es: la capacidad de los puertos con respecto al calado que utilizan puede ser modificado para que se puedan comercializar más productos; además de esto, la infraestructura de los puertos puede ser buena en general, pero la capacidad de entrada de buques es muy inferior con respecto a lo que se podría obtener de estas zonas de comercio marítimo, la entrada de buques más grande ampliaría la capacidad de comercialización que tiene el país, haciéndolo más competente.

El segundo factor es el acceso por carretera y la saturación de espacios, lo cual impide que el proceso sea ágil, retrasando los demás procedimientos y generando un aumento considerable en los costos, lo cual hace que las dinámicas comerciales no sean tan atractivas debido a su costo.

Las problemáticas del puerto también tienen un panorama social bastante profundo, ya que la región sufre violencia, narcotráfico y falta de intervención estatal, esto no permite una correcta adecuación de las vías de transporte internas desde el puerto hacia el resto de país, aumentando los costos y los tiempos de despacho de mercancías.

Como fue posible observar en la tabla comparativa entre los puertos 
de Cartagena y Buenaventura, el puerto de Cartagena cuenta con mejor capacidad de calado y sus tiempos de despacho de mercancía son menores con respecto al puerto de Buenaventura; sin embargo, ambos puertos son los más importantes del país y el puerto de buenaventura es el único en la región pacifica, por lo cual es necesario implementar nuevas medidas para iniciar procesos de dragado que permitan la entrada a buques con mayor capacidad de carga, así como mejora en la logística interna del puerto para reducir los tiempos de despacho y recepción de mercancías.

Colombia tiene muchos retos por delante. Es necesario gestionar nuevos proyectos no solo para la mejora infraestructural, sino para aplacar las problemáticas regionales y fomentar el desarrollo de las zonas en su totalidad, desarrollo entendido en términos comerciales, infraestructurales y sociales.

\section{REFERENCIAS}

Banco de La Republica. (2014). De la Sustitución de Importaciones. Recuperado dehttp://www.banrepcultural.org/blaavirtual/economia/industrilatina/003.htm

CEPAL. (2014). Perfil Maritimo y Logistico de America Latina y el Caribe. Recuperado de http://
www.cepal.org/cgi-bin/getProd. asp? $\mathrm{xml}=/$ perfil $/$ noticias $/$ noticias/4/54974/P54974.xml\&xsl=/ perfil/tpl/p1f.xsl\&base=/perfil/ tp1/top-bottom.xsl

Datosmacro. (s.f.). Recuperado de http://www.datosmacro.com/

Departamento Nacional de Planeación. (20 de Diciembre de 2001). Ducumento CONPES 3149. Recuperado de https://www.mintransporte.gov.co/descargar. php?id=473

Doing Business. (s.f.). Clasificación de las economías. Recuperado de http://www.doingbusiness.org/ Grupo de Planificación Sectorial. (2011). Ministerio de Transporte. Recuperado de https://www. mintransporte.gov.co/Documentos/documentos del ministerio/ Estadisticas

Johnson, J., Wood, D., Wardlow, D., \& Murphy, P. (1999). Contemporary logistics. New Jersey: Pretince Hall.

Ministerio de Industria y Turismo. (2014). Acuerdos Comerciales Firmados por Colombia TLCS. Recuperado de http://www.min cit.gov.co/tlc/publicaciones. php?id=11951

Ministerio de Transporte. (2014). Superintendencia de Puertos $y$ Transporte. Recuperado de http://www.supertransporte. gov.co/index.php/la-entidad/ historia.html 
Morato, Y. (julio de 2010). Superintendencia de Puertos y Transporte. Recuperado de http:// www.supertransporte.gov.co/ documentos/2014/delegada $\% 20$ puertos/caracterizacion $\% 20$ puertos/LOGISTICA\%20PORTUARIA.pdf

Navickas, V., Sujeta, L., \& Vojtovich, S. (2011). Logistics Systems as a Factor of Country's Competitiveness. Economics and Management, 16, 231-237.

Pérez, G.J. (Octubre de 2005). Banco de la República. Recuperado de http://www.banrep.gov.co/ docum/Lectura_finanzas/pdf/ DTSER-64.pdf

Oficina Asesora de Planeación.(2014). MinTransporte. Recuperado de https://www.mintransporte.gov. co/Documentos/documentos del ministerio/Estadisticas

Procolombia. (2015). procolombia. Recuperado de www.procolombia.co [consultado diciembre 15 de 2015]

Real Academia Española. Diccionario de la lengua española. (s.f.). Recuperado de http://dle.rae. es/?id=LYf3Ibz

Rendón, G.A. (4 de Junio de 2009). Desarrollo y transformación de los puertos en Colombia. Un enfoque desde las competencias laborales. Recuperado de http:// publicaciones.unisimonbolivar.edu.co/rdigital/educacion/ index.php/educacion/article/ viewFile/14/13

Sociedad Portuaria De Cartagena $S . A$. (s.f.). SPRC. Recuperado de http://cisne.puertocartagena. com/

Sociedad Portuaria Regional de Buenaventura. (s.f.). SPRBUN. Recuperado de http://www.sprbun. com/

\section{Anexo 1. Glosario}

- Calado: Profundidad que alcanza en el agua la parte sumergida de una embarcación.

- Dragado: operación de limpieza de rocas y sedimentos en cursos de agua, lagos, bahías y accesos a puertos para aumentar la profundidad de un canal.

- Escáner: Para el funcionamiento portuario, se trata de una máquina conectada a una red de comunicaciones que permite, mediante un programa, analizar el estado de los puertos. ${ }^{2}$

- Granel: Se aplica al producto que se vende sin envasar o sin empaquetar, o a la manera de comprar o vender productos de este tipo.

2 Definición propia, basada en diferentes descripciones del producto. 
- Grúa pórtico: Son grúas especializadas en la carga y manejo de contenedores. ${ }^{3}$

- Infraestructura: Conjunto de elementos, dotaciones o servicios necesarios para el buen funcionamiento de un país, de una ciudado de una organización cualquiera. ${ }^{4}$

- Patios: Espacios destinados para el almacenamiento de contenedores. $^{5}$

- Plataformas: Las plataformas marítimas son estructuras construidas sobre el nivel del mar que facilitan las diferentes operaciones portuarias. ${ }^{6}$
- Rata de cargue: Velocidad con la que se embarca. ${ }^{7}$

- Reach stackers: Vehículos para la manipulación de contenedores. $^{8}$

- Sedimentación: Depósito o acumulación de materiales arrastrados mecánicamente por las aguas o el viento.

- Transtainer: Son grúas empleadas para la carga y descarga de contenedores sobre camiones, vagones de tren y/o poner contenedores uno encima de otro. ${ }^{9} 10$

3 Definición propia basada en (Morato, 2010). Ver referencias bibliográficas.

4 Bajo esta definición se realizará el análisis.

5 Definición propia basada en (Morato, 2010). Ver referencias bibliográficas.

6 Definición propia basada en (Morato, 2010). Ver referencias bibliográficas.

7 Definición propia basada en (Morato, 2010). Ver referencias bibliográficas.

8 Definición propia, basada en las descripciones de la máquina presentada por los diferentes oferentes del producto.

9 Definición propia, basada en diferentes descripciones del producto.

10 Las demás definiciones son copiadas del diccionario de la lengua española de la Real Academia Española. 